\title{
Phosphate-solubilizing fungi isolated from a semiarid area cultivated with melon (Cucumis melo L. cv. gold mine)
}

Flavia Paiva Coutinho ${ }^{1,3}$, Maria Auxiliadora de Queiroz Cavalcanti ${ }^{1}$ and Adriana Mayumi Yano-Melo ${ }^{2}$

Recebido em 21/10/2008. Aceito em 9/09/2011

RESUMO

(Fungos solubilizadores de fosfato isolados de área semiárida cultivada com melão (Cucumis melo L. cv. gold mine). Considerando que pouco se conhece sobre a ocorrência de fungos solubilizadores de fosfato de áreas cultivadas com melão, foi avaliada a habilidade de solubilização desse nutriente por fungos filamentosos isolados dessas áreas. Foram avaliadas 318 amostras de fungos filamentosos pertencentes a 23 gêneros, além de Aphyllophorales e Mycelia sterilia. Dessas amostras, 52 apresentaram habilidade para solubilizar o fosfato: Aphyllophorales (2), Aspergillus (34), Penicillium (10) e Rhizopus (6). Esses resultados contribuem para subsidiar pesquisas que testem a capacidade desses fungos em solubilizar outras fontes fosfatadas aplicadas na cultura do melão, assim como indicam a necessidade de selecionar isolados com maior capacidade e potencial para solubilização.

Palavras-chave: adubação, fungos filamentosos, micro-organismos, Vale do São Francisco

\begin{abstract}
(Phosphate-solubilizing fungi isolated from a semiarid area cultivated with melon (Cucumis melo L. cv. gold mine). Considering that little is known about the occurrence of phosphate-solubilizing fungi from areas cultivated with melon, the phosphate solubilization ability of filamentous fungi isolated in these areas was evaluated. Three hundred and eighteen filamentous fungal isolates belonging to 23 genera were evaluated, besides Aphyllophorales and Mycelia sterilia. From those, 52 were able to solubilize P: Aphyllophorales (2), Aspergillus (34), Penicillium (10) and Rhizopus (6). These results will contribute to subsidizing further research regarding the capacity of these fungi to solubilize other sources of phosphate applied to the melon crop, as well as indicate the need for a screening program to select those with higher capacity and potential for solubilization.
\end{abstract}

Key words: fertilization, filamentous fungi, microorganism, San Francisco Valley

Melon is one of the most demanding cucurbitaceous crops, usually demanding potassium, nitrogen and phosphorus fertilization (Vitti et al. 1994). Phosphorus stimulates growth and is essential to root formation, flowering and fructification, acting as a decisive factor in the quality and quantity of fruits (Crisóstomo et al. 2002).

Plant productivity is often limited by low levels of soil nutrients in absorbable forms. Phosphorus is possibly the most limiting nutrient in tropical regions, considering its high stability and, consequently, low solubility (Moreira \& Siqueira 2006). Microbes have the ability to make insoluble $P$ available by the processes of solubilization or mineralization. The solubilization process occurs through the production of organic acids that directly dissolve phosphate minerals or by a chelating action over soil cations liberating soluble phosphates (Silva
Filho et al. 2002). Therefore, P solubilizing microorganisms play a key role in the biogeochemical cycle of phosphorus and its availability to plants (Carneiro et al. 2004).

With the development of organic agriculture in fruit production in the San Francisco Valley, many organic residues have been used as fertilizers. However, little is known about the effect of these residues on the soil mycobiota, especially on phosphate-solubilizing fungi. Organic residues that favor the increase of the P-solubilizing fungal population are of utmost importance for improving the nutritional condition of the crops in the region. The aim of this study was to evaluate the ability of phosphate solubilization by filamentous fungi isolated from the rhizosphere of melon plants (Cucumis melo L. cv. gold mine) fertilized with organic composts.

\footnotetext{
${ }^{1}$ Universidade Federal de Pernambuco, Centro de Ciências Biológicas, Departamento de Micologia, Recife, PE, Brasil

${ }^{2}$ Universidade Federal do Vale do São Francisco, Campus de Ciências Agrárias, Colegiado de Zootecnia, Petrolina, PE, Brasil

${ }^{3}$ Author for correspondence: flaviapaco@hotmail.com
} 
Table 1. Filamentous fungi evaluated for the ability to solubilize phosphorus.

\begin{tabular}{|c|c|c|c|c|c|}
\hline TAXA & No of samples & PSF & TAXA & No of samples & PSF \\
\hline Alternaria tenuissima (Kunze) Wiltshire & 01 & - & Humicola fuscoatra Traaen & 07 & - \\
\hline Aspergillus flavipes (Bainier \& R. Sartory) Thom \& Church & 02 & - & Monodictys castaneae (Wallr.) S. Hughes & 02 & - \\
\hline A. flavus Link & 02 & + & Myrothecium indicum P.Rama Rao & 03 & - \\
\hline A. fumigatus Fresenius & 18 & - & M. roridum Tode & 03 & - \\
\hline A. japonicus var. aculeatus (Iizuka) Al-Musallam & 03 & + & M. verrucaria (Alb. \& Schwein.) Ditmar & 03 & - \\
\hline A. japonicus var. japonicus Saito & 08 & + & $\begin{array}{l}\text { Neocosmospora vasinfecta var. africana (Arx) } \\
\text { P.F. Cannon \& D. Hawksw. }\end{array}$ & 11 & - \\
\hline A. nidulans (Eidam) G. Winter & 03 & - & $\begin{array}{l}\text { Paecilomyces carneus (Duché \& R. Heim) } \\
\text { A.H.S. Br. \& G. Sm. }\end{array}$ & 01 & - \\
\hline A. niger Tieghem & 16 & + & P. lilacinus (Thom) Samson & 02 & - \\
\hline A. niger var. niger Tieghem & 02 & + & P. variotii Bainier & 01 & - \\
\hline A. sydowii (Bainier \& Sartory) Thom \& Church & 01 & - & Penicillium citreonigrum Dierckx & 01 & + \\
\hline A. tamarii Kita & 03 & + & P. citrinum Thom & 04 & - \\
\hline A. terreus Thom & 17 & - & P. corylophilum Dierckx & 02 & - \\
\hline A. terreus var. aureus Thom \& Raper & 09 & - & P. crustosum Thom & 01 & + \\
\hline A. ungüis (Weill \& L. Gaudin) Thom \& Raper & 05 & - & P. decumbens Thom & 04 & + \\
\hline A. ustus (Bainier) Thom \& Church & 02 & - & P. fellutanum Biourge & 03 & - \\
\hline A. viridinutans Ducker \& Thrower & 05 & - & P. griseofulvum Dierckx & 03 & - \\
\hline Chaetomium convolutum Chivers & 03 & - & P. janthinellum Biourge & 04 & + \\
\hline C. cupreum L.M. Ames & 01 & - & P. pinophilum Thom & 02 & - \\
\hline C. leucophora L.M. Ames & 01 & - & P. restrictum J.C. Gilman \& E.V.Abbott & 05 & - \\
\hline C. nigricolor L.M. Ames & 02 & - & P. spinulosum Thom & 01 & - \\
\hline C. ochraceum Tschudy & 03 & - & P. vinaceum J.C. Gilman \& E.V. Abbott & 07 & - \\
\hline C. trigonosporum (Marchal \& É.J. Marchal) Chivers & 05 & - & P. waksmanii K.M. Zalessky & 01 & - \\
\hline Cladosporium sphaerospermum Penz. & 02 & - & $\begin{array}{l}\text { Rhizopus microsporus var. chinensis (Saito) } \\
\text { Schipper \& Stalpers }\end{array}$ & 06 & + \\
\hline C. tenuissimum Cooke & 02 & - & R. microsporus var. microsporus Tieghem & 07 & - \\
\hline Curvularia eragrostidis (Henn.) J.A. Mey. & 01 & - & Scopulariopsis brumptii Salv.-Duval & 01 & - \\
\hline Emericella nidulans (Eidam) Vuill. & 15 & - & S. croci J.F.H. Beyma & 01 & - \\
\hline E. nidulans var. acristata Subramaniam & 17 & - & S. sphaerospora Zach & 04 & - \\
\hline E. nidulans var. echinulata Godeas & 09 & - & Scytalidium lignicola Pesante & 01 & - \\
\hline E. rugulosa (Thom \& Raper) C.R. Benj. & 01 & - & $\begin{array}{l}\text { Sordaria fimicola (Roberge ex Desm.) } \\
\text { Ces. \& De Not. }\end{array}$ & 02 & - \\
\hline E. variecolor Berk. \& Broome & 01 & - & $\begin{array}{l}\text { Talaromyces trachyspermus (Shear) } \\
\text { Stolk \& Samson }\end{array}$ & 02 & - \\
\hline $\begin{array}{l}\text { Eupenicillium brefeldianum (B.O. Dodge) Stolk \& D.B. } \\
\text { Scott }\end{array}$ & 01 & - & Thielavia fragilis (Natarajan) Arx & 04 & - \\
\hline E. crustaceum F. Ludw. & 01 & - & T. microspora Mouch. & 03 & - \\
\hline Eurotium chevalieri L. Mangin & 04 & - & T. terrestris (Apinis) Malloch \& Cain & 01 & - \\
\hline E. rubrum W. Bremer & 01 & - & $\begin{array}{l}\text { T. terricola (J.C. Gilman \& E.V. Abbott) } \\
\text { C.W. Emmons }\end{array}$ & 17 & - \\
\hline Fusarium equiseti (Corda) Sacc. & 01 & - & $\begin{array}{l}\text { Torula caligans (Bat. \& H.P. Upadhyay) } \\
\text { M.B. Ellis }\end{array}$ & 07 & - \\
\hline F. merismoides Corda & 02 & - & Trichoderma pseudokoningii Rifai & 01 & - \\
\hline F. oxysporum E.F. Sm. \& Swingle & 03 & - & T. virens (J.H. Mill., Giddens \& A.A. Foster) Arx & 01 & - \\
\hline F. redolens Wollenw. & 01 & - & T. viride Pers. & 01 & - \\
\hline F. solani (Mart.) Saccardo & 09 & - & Aphyllophorales & 02 & + \\
\hline Fusarium stilboides Wollenw. & 02 & - & Black Mycelia sterilia & 01 & - \\
\hline TOTAL & 185 & & & 133 & \\
\hline
\end{tabular}

PSF $=$ Phosphate-solubilizing fungi. 
Fifty-four rhizosphere soil samples were collected at random from an irrigated semiarid area cultivated with melon (C. melo cv. gold mine) in the San Francisco Valley, Petrolina, Brazil (09²'09' 'S, 4055'28' 'W). Aliquots (1 mL) of serial dilutions (1:1000) of the soil samples were plated on Sabouraud Agar medium with chloranphenicol (500 $\left.\mathrm{mg} \mathrm{l}^{-1}\right)$, and incubated at $28^{\circ} \mathrm{C}$ for up to $96 \mathrm{~h}$. The fungal colonies were transferred and maintained on Potato Dextrose Agar (PDA). Fungal species were identified based on colony morphology on different media (Czapeck, malt extract agar and PDA) and micromorphological characteristics. The evaluation of the ability of these fungi to solubilize phosphorus was carried out by removing a $5 \mathrm{~mm}$ diameter disc from the edge of the colony and transferring it to a Petri dish containing GAGES solid medium (glucose, arabinosis, glycerol, soil extract, $\mathrm{CaCl}_{2}, \mathrm{MgSO}_{4}, \mathrm{NaCl}$ and agar) supplemented with $10 \% \mathrm{~K}_{2} \mathrm{HPO}_{4}(50 \mathrm{ml})$ and $10 \% \mathrm{CaCl}_{2}(50 \mathrm{ml})$, to produce inorganic phosphate precipitate, $\mathrm{CaHPO}_{4}$ (Souchie et al. 2006). The plates were kept at room temperature $\left(28^{\circ} \mathrm{C}\right)$ for 12 days and the colonies that formed a clear zone around them, due to solubilization of $\mathrm{CaHPO}_{4}$, were considered P-solubilizing fungi.

From the soil samples, 318 filamentous fungi (Tab. 1) were isolated, belonging to 23 genera and 78 species, besides Aphyllophorales and Mycelia sterilia. Fifty-two of them were able to solubilize P, as follows: Aspergillus flavus (2), A. japonicus var. aculeatus $^{*}$ (3), A. japonicus var. japonicus ${ }^{\star}(8)$, A. niger (16), A. niger var. niger ${ }^{\star}(2), A$. tamarii ${ }^{\star}(3)$, Penicillium citreonigrum $^{\star}$ (1), . crustosum $^{\star}(1)$, P. decumbens ${ }^{\star}(4)$, P. janthinellum ${ }^{\star}(4)$, Rhizopus microsporus var. chinensis ${ }^{\star}(6)$ and Aphyllophorales (2). Those marked $\left(^{\star}\right)$ are reported for the first time as P-solubilizers.

Among the identified species, $15 \%$ are P-solubilizers, which agrees with values usually found soil (8 to 58\% of all fungi), as reported by Nahas et al. (1994). Aspergillus and Penicillium species stood out as phosphorus solubilizers, and these two genera are the most cited in the literature with this capability (Silva Filho et al. 2002; Wakelin et al. 2004; Souchie et al. 2006). However, most works have not identified the species, making comparisons only possible at the genus level. All 16 samples of $A$. niger were able to solubilize phosphate. This species has been isolated from soils cultivated with a wide variety of plants, such as coconut, beets, olive, wheat, chickpea, soybean, broad bean, rice, sugar cane, Pinus, Eucalyptus and Avicennia germinans (Wahid \& Mehana 2000; Abd-Alla \& Omar 2001; Rashid et al. 2004; Zayed \& AbdelMotaal 2005). Two samples of Aspergillus flavus solubilized $\mathrm{P}$, which has been reported for the species (Abd-Alla \& Omar 2001; Sapatnekar et al. 2003; Rashid et al. 2004). Aspergillus fumigatus, A. nidulans, A. terreus, Emericella nidulans, Fusarium oxysporum, Sordaria fimicola and Thielavia terricola were not able to solubilize $\mathrm{P}$, but other isolates of the same species from Indian soils were reported as phosphorus solubilizers (Sapatnekar et al. 2003). Similarly, Penicillium citrinum and Trichoderma viride did not show the capacity to solubilize $\mathrm{P}$ in this study, although P. citrinum, isolated from wheat, soybean and broad bean rhizospheres (Abd-Alla \& Omar 2001), and T. viride, isolated from black soil of Maharashtra, India, and sugar cane plantations (Sapatnekar et al. 2003; Zayed \& Abdel-Motaal 2005), have been reported as solubilizers. A screening program to select those isolates with high capacity and potential for solubilization is planned, with the further goal of a program for controlled inoculation.

\section{Acknowledgements}

The authors would like to thank the CAPES, CNPq and Embrapa Semiárido for supporting the project and Dra. Elaine Malosso and Leonardo Costa for reviewing the English text.

\section{References}

Abd-Alla, M.H. \& Omar, S.A. 2001. Survival of Rhizobia/Bradyrhizobia and a rock-phosphate-solubilizing fungus Aspergillus niger on various carriers from some agro-industrial wasters and their effects on nodulation and growth of faba bean and soybean. Journal of Plant Nutrition 24: 261-272.

Carneiro, R.G.; Mendes, I.C.; Lovato, P.E.; Carvalho, A.M. \& Vivaldi, L.J. 2004. Indicadores biológicos associados ao ciclo do fósforo em solos de Cerrado sob plantio direto e plantio convencional. Pesquisa Agropecuária Brasileira 39: 661-669.

Crisóstomo, L.A.; Santos, A.A.; Van Raiji, B.; Faria, C.M.B.; Silva, D.J.; Fernandez, F.A.M.; Santos, F.J.S.; Crisóstomo, J.R.; Freitas, J.A.D.; Holanda, J.S.; Cardoso, J.W. \& Costa, N.D. 2002. Adubação, irrigação, híbridos e práticas culturais para o meloeiro no Nordeste. Fortaleza, Ministério da Agricultura, Pecuária e Abastecimento.

Moreira, F.M.S. \& Siqueira, J.O. 2006. Microbiologia e bioquímica do solo. Lavras, Editora UFLA.

Nahas, E.; Centurion, J.F. \& Assis, L.C. 1994. Microrganismos solubilizadores de fosfato e produtores de fosfatases de vários solos. Revista Brasileira de Ciência do Solo 18: 43-48.

Rashid, M.; Khalil, S.; Ayub, N.; Alam, S. \& Latif, F. 2004. Organic acids production and phosphate solubilization by phosphate solubilizing microorganisms (PSM) under in vitro conditions. Pakistan Journal of Biological Sciences 7: 187-196.

Sapatnekar, H.G.; Rasal, P.H. \& Patil, P.L. 2003. Occurrence of phosphate solubilizing fungi in black soils of Maharashtra. Journal of Maharashtra Agricultural Universities 28: 164-165.

Silva Filho, G.N; Narloch, C. \& Scharf, R. 2002. Solubilização de fosfatos naturais por microrganismos isolados de cultivos de Pinus e Eucalyptus de Santa Catarina. Pesquisa Agropecuária Brasileira 37: 847-854.

Souchie, E.L.; Saggin-Júnior, O.J.; Silva, E.M.R.; Campello, E.F.C.; Azcón, R. \& Barea, J.M. 2006. Communities of P-solubilizing bacteria, fungi and arbuscular mycorrhizal fungi in grass pasture and secondary forest of Paraty, RJ - Brazil. Anais da Academia Brasileira de Ciências 78: 183-193.

Vitti, G.C.; Holanda, J.S. de; Hernandez, F.B.T.; Boaretto, A.E. \& Penteado, S.R. 1994. Fertirrigação: condições e manejo. Pp. 195264. In: Anais da XXI Reunião Brasileira de Fertilidade do Solo e Nutrição de Plantas. Petrolina 1994. Viçosa, Sociedade Brasileira de Ciência do Solo.

Wahid, O.A. \& Mehana, T.A. 2000. Impact of phosphate-solubilizing fungi on the yield and phosphorus-uptake by wheat and faba bean plants. Microbiology Research 155: 221-227.

Wakelin, S.A.; Warren, R.A.; Harvey, P.R. \& Ryder, M.H. 2004. Phosphate solubilization by Penicillium spp. closely associated with wheat roots. Biology and Fertility of Soils 40: 36-43.

Zayed, G. \& Abdel-Motaal, H. 2005. Bio-production of compost with low $\mathrm{pH}$ and high soluble phosphorus from sugar cane bagasse enriched with rock phosphate. World Journal of Microbiology and Biotechnology 21: 747-752. 